\title{
Aetiology, management and strategies for prevention of postoperative respiratory failure (PORF) after thoracic surgery
}

\author{
Marcus Taylor ${ }^{1}$, Mike Charlesworth ${ }^{2}$, Tim Hayes ${ }^{2}$, Vijay Joshi ${ }^{1}$ \\ ${ }^{1}$ Department of Cardiothoracic Surgery, ${ }^{2}$ Department of Cardiothoracic Anaesthesia, Wythenshawe Hospital, Manchester University NHS \\ Foundation Trust, Manchester, UK \\ Contributions: (I) Conception and design: All authors; (II) Administrative support: M Taylor; (III) Provision of study materials or patients: M Taylor; \\ (IV) Collection and assembly of data: M Taylor; (V) Data analysis and interpretation: All authors; (VI) Manuscript writing: All authors; (VII) Final \\ approval of manuscript: All authors. \\ Correspondence to: Marcus Taylor, MRCS. Department of Cardiothoracic Surgery, Wythenshawe Hospital, Manchester University NHS Foundation \\ Trust, Southmoor Road, Manchester, M23 9LT, UK. Email: Marcus.taylor1@nhs.net.
}

\begin{abstract}
Post-operative pulmonary complications (PPCs) occur in a large proportion of patients after thoracic surgery and are associated with higher rates of morbidity and mortality. Avoidance of PPCs can be achieved through a multi-disciplinary and multifactorial approach which addresses a number of preoperative, intra-operative and post-operative factors. A subgroup of patients who develop PPCs will progress to post-operative respiratory failure (PORF). Treatment strategies for these patients all have the underlying aim of avoiding the need for re-intubation, which is associated with extremely high mortality. This review considers the aetiology, management and strategies for prevention of PORF after thoracic surgery.
\end{abstract}

Keywords: Complications; respiratory failure; postoperative; thoracic surgery

Received: 14 February 2020; Accepted: 23 March 2020; Published: 10 January 2021.

doi: $10.21037 /$ shc.2020.03.10

View this article at: http://dx.doi.org/10.21037/shc.2020.03.10

\section{Background}

Lung cancer is the third most common cancer in the UK with over 47,000 new diagnoses each year. The National Cancer Registration and Analysis Service has quoted that the proportion of patients with non-small cell lung cancer (NSCLC) undergoing surgery each year in the UK was approximately $16 \%$ between 2013 and 2015, which represents a marked increase from the $8.8 \%$ and $10.6 \%$ quoted for 1998 and 2008 respectively (1). Following surgery for lung cancer, the 30 -day mortality rate is approximately $2 \%$, and rises to around $5 \%$ at 90 days. Oneyear survival after lung cancer surgery is around $88 \%$ (2).

Reduction of mortality and morbidity after lung cancer surgery is as an important component of improving lung cancer survival. This is because most patients undergoing surgery are early stage (stage I and II) lung cancer patients. Hence, if survival in this group of patients can be optimised, it is likely to have a significant (and disproportionate)

(c) Shanghai Chest. All rights reserved. impact on overall lung cancer survival rates, because if these patients survive the perioperative period they are much more likely to survive to five years and beyond as compared with patients with more advanced lung cancer.

\section{Complications after thoracic surgery}

Development of post-operative complications is associated with poor patient satisfaction (3), prolonged critical care and overall hospital stay, increased healthcare costs and increased mortality. Post-operative complications after thoracic surgery occur in around $15-40 \%$ of patients (4). Moreover, the incidence of even a single post-operative complication can lead to additional adverse outcomes (5). Studies looking to reduce rates of complications in this patient group have identified several risk factors associated with complications after thoracic surgery. Interventions aimed at modifying these risk factors could therefore logically lead to better outcomes and improved survival 
after surgery (6-8).

Whilst cardiovascular complications such as atrial fibrillation (which has a reported incidence of between $4 \%$ and $37 \%$ ), myocardial infarction, and renal complications (primarily acute kidney injury), are recognised as commonly-occurring complications after thoracic surgery (9-11), the majority of complications in this patient population are defined as postoperative pulmonary complications (PPCs).

\section{PPCs}

The term 'post-operative pulmonary complication' is broad and encompasses many different pathologies including prolonged air leak, pleural effusion, pneumothorax, atelectasis/lobar collapse, pneumonia, pulmonary embolus, pulmonary oedema and acute lung injury, including acute respiratory distress syndrome (ARDS). A recent systematic review provided consensus definitions for PPCs. The definition of PPCs was based on the mechanism (atelectasis, pneumonia, ARDS or aspiration) and the severity (based on oxygen requirement and/or need for ventilation) (12). The incidence of PPCs is high, ranging from 14\% to $37 \%$, since thoracic surgery impairs postoperative respiratory function in a group of patients with a high prevalence of chronic lung disease. Indeed, some reports in the literature state that PPCs are responsible for over $80 \%$ of deaths after thoracic surgery (6). Development of a PPC is multifactorial and a number of patient, surgical and anaesthetic factors have been proven to be influential in the development of PPCs.

\section{Postoperative respiratory failure (PORF)}

According to guidelines issued by a European taskforce on perioperative clinical outcome definitions, the term PORF is defined as "post-operative $\mathrm{PaO}_{2}<8 \mathrm{kPa}$ on room air, a $\mathrm{PaO}_{2}: \mathrm{FiO}_{2}$ ratio $<40 \mathrm{kPa}$ or arterial oxybaemoglobin saturation measured with pulse oximetry $<90 \%$ and requiring oxygen therapy". When using this definition, a significant proportion of PPCs recorded after thoracic surgery would be classified as respiratory failure.

Other definitions in common use include "mechanical ventilation for $>24$ bours after surgery or unplanned reintubation due to respiratory causes" (13). In the most severe cases of PORF, admission (or re-admission) to critical care is required for mechanical ventilation (and often subsequent tracheostomy) or veno-venous extracorporeal membrane oxygenation ( $\mathrm{V}$-ECMO) to be provided.
Hence 'respiratory failure' should be recognised as a term which encompasses the more serious end of the spectrum of postoperative pulmonary pathology. Therefore, by definition, the incidence of PORF can be reduced by targeting a reduction in the overall incidence of PPCs.

The incidence of PORF requiring some form of mechanical intervention after thoracic surgery has been reported to be approximately $1 \%$, further increasing to $3-5 \%$ in the high-risk patient population and is associated with a sharp increase in both morbidity and mortality (14). In addition to the effect on physical health, unplanned critical care admission is perceived as a particularly deleterious event by patients, as it clearly represents a move backwards, in terms of progress from surgery to discharge. This perception is supported by national data, which demonstrates a significantly higher length of stay and rate of adverse outcomes in those patients requiring an unplanned admission to a critical care environment (15). The CALoR 1 trial, which looked specifically at unplanned admission to critical care after lung resection found that mortality was significantly higher for those patients requiring unplanned critical care admission ( $0.2 \%$ vs. $26.7 \%, \mathrm{P}<0.001)$. Although this was a single-centre trial the cohort was sizeable $(1,169$ patients). Based on the results of this pilot study the Association of Cardiothoracic Anaesthetists (ACTA) have commissioned a multi-centre study to assess whether these findings are replicated in the wider population of patients undergoing lung resection (16).

\section{Enhanced recovery pathways}

Over the past two decades there has been a radical shift towards implementing enhanced recovery protocols for patients undergoing major surgery following the publication of high-quality evidence demonstrating the efficacy of the programme in reducing postoperative length of stay and complications, particularly in colorectal surgery (17). A 2013 systematic review of aspects of perioperative care for thoracic surgery patients that could be incorporated into an enhanced recovery programme was one of the first UK-based studies to address this topic (18). Subsequently, the European Society of Thoracic Surgeons (ESTS), in conjunction with the Enhanced Recovery After Surgery Society (ERASS), published updated guidance in 2019 regarding the implementation of enhanced recovery protocols in thoracic surgery patients and made 45 recommendations across the pre-operative, admission, perioperative and postoperative phases of the patient journey (19). 


\section{Pre-operative strategies for prevention of PPCs}

There are two key areas of focus during the pre-operative period for prevention of PPCs. The first is centred around patient selection, to ensure that patients whose risk profile renders surgery an inappropriate treatment option, are reliably identified during this period. The second component of pre-operative PPC prevention is the identification and optimisation of modifiable risk factors, including smoking, alcohol intake and poor nutrition.

\section{The impact of surgical volume}

The effect of hospital and surgeon volume on outcomes has also been studied. Whilst there is very little available data focussing specifically on PPCs, an article by Al-Sahaf $e t$ al. reviewed a number of studies which addressed the impact of volume on short-term mortality and overall survival after thoracic surgery (20). Indeed, they mentioned numerous studies from the UK, Europe and the USA, all of which demonstrated that a higher centre volume was associated with better outcomes after surgery. Associations between individual surgeon volume and outcomes were much more equivocal. Of interest was a UK-based study comprised of 12,862 patients, which demonstrated that patients who were older and had a higher number of co-morbidities were more likely to receive surgery in high-volume centres (defined as those carrying out more than 70 resections per year). Despite this more permissive approach to surgical selection, the study confirmed that patients in the higher volume centres had much better overall outcomes, and that this survival advantage was at its most pronounced in the early post-operative period (21).

\section{Pre-operative identification of the high-risk patient}

High quality patient selection is vital, to ensure that preoperative decision-making is robust and reproducible. Riskstratification models, such as Thoracoscore for thoracic surgery, are used as aids to augment the decision-making process. However, it is well recognised that Thoracoscore does not predict mortality equally accurately across all patient groups (22), perhaps as a result of changing patient characteristics since its inception, and hence the predictions made by these tools must be considered alongside other components of the pre-operative decision-making process, such as objective functional testing and multidisciplinary team meetings convened specifically to discuss high-risk patients (23). Final decisions regarding treatment should be made on an individual case by case basis.

\section{Pulmonary function tests and objective functional testing}

Both pulmonary function tests and objective functional tests are frequently used to aid with stratification of risk. The role of pre-operative pulmonary function tests (particularly FEV1) in predicting outcomes in thoracic surgery patients has been studied in detail. Many studies have found that FEV1 and predicted postoperative FEV1 values were significant independent factors on univariate analysis but not on multivariate analysis. It has been postulated that better patient selection now means that fewer patients with poor pulmonary function tests are undergoing surgery, meaning that FEV1 is no longer such an important factor (24). Furthermore, increasing amounts of research are being devoted to gas transfer, which is emerging as a more effective predictor of outcomes in thoracic surgery patients $(25,26)$

As technology has developed, more and more focus has been placed on objectively measuring pre-operative mobility and its effects on outcomes. Pre-operatively in thoracic surgery, this manifests through several objective functional tests which are performed in addition to pulmonary function tests, (particularly in those with poor or borderline pulmonary function tests) to augment the decision-making process in terms of assessing suitability for surgery. These functional tests include the six-minute walk, the shuttle test and the cardiopulmonary exercise test (CPET) and have been proven to correlate well with postoperative outcomes (27-30).

\section{Risk factors for PPCs after thoracic surgery}

The significant impact of PPCs on outcomes after thoracic surgery has led to multiple studies setting out to identify specific risk factors that predispose patients to developing PPCs. However, many of these studies are retrospective and dependent upon the quality of data collected, with flaws such as missing data from surgical databases and nonrecording of perceived 'minor' complications noted in certain studies. Moreover, many risk factors are interrelated (i.e., smoking, poor pulmonary function tests and chronic obstructive pulmonary disease). Furthermore, prohibitively small sample sizes prevent multivariable analyses from being performed in order to identify truly independent 
risk factors (31,32). Latterly, the importance of focussing on modifiable (in contrast to non-modifiable) risk factors has found favour as a potentially effective method of reducing the incidence of post-operative complications (33). The risk factors most commonly identified from multiple studies as strongly associated with PPCs include advanced age, poor pre-operative pulmonary functions tests, smoking status, cardiovascular comorbidities, chronic obstructive pulmonary disease and baseline functional status $(24,31,32,34,35)$. Modifiable risk factors amenable to pre-operative optimisation include anaemia, malnutrition, smoking and excess alcohol intake.

\section{Modifiable risk factors: smoking cessation}

Current smokers have been found to be twice as likely to develop PPCs in comparison to never smokers and exsmokers of more than four weeks (36). Some studies have found recent ex-smokers (smoking cessation less than four weeks prior to surgery) maintain their increased risk of PPCs but delaying surgery in order to extend the smokingfree period should be carefully considered on a caseby-case basis due to the possibility of cancer upstaging and its impact on long-term survival. Unfortunately, current evidence suggests that the success rate of smoking cessation in this patient group remains low. Despite this, as a modifiable risk factor strongly associated with the development of PPCs, smoking cessation should remain a key focus for pre-operative optimisation of patients undergoing thoracic surgery.

\section{Modifiable risk factors: alcohol excess}

The systemic effects of chronic excessive alcohol consumption on the liver, pancreas, cardiovascular system, neurological system, clotting cascade and immune function are well recognised. When focussing specifically on a population of patients undergoing surgery for lung cancer, alcohol abuse has been regularly identified as a factor associated with adverse outcomes, and with PPCs specifically $(37,38)$. However, whilst cessation of alcohol prior to surgery is recommended, there is no good evidence outlining the optimal alcohol-free period prior to surgery (19).

\section{Pre-operative cardiopulmonary rehabilitation}

Poor pre-operative exercise capacity is associated with an increased risk of PPCs. In recent years, in conjunction with the increased popularity of ERAS, the concept of 'prehabilitation' has been established. Focussing on augmenting physiological reserve and its subsequent effect on functional capacity, there is high quality evidence available (in the form of a meta-analysis and Cochrane review) examining whether prehabilitation in thoracic surgery affects outcomes. Although the studies in this area are heterogeneous in nature, meaning that the optimal prehabilitation programme structure is not clear, the metaanalysis demonstrated a clear reduction in the incidence of PPCs amongst patients that underwent pre-operative optimisation in the form of prehabilitation (39). However, implementing these approaches is often resource-heavy. Moreover, in this patient population, where the time from referral to surgery is often very short, the opportunity to undertake a meaningful period of prehabilitation is limited.

\section{Intra-operative factors}

Once a patient has been appropriately selected for surgery and their risk factors adequately optimised, there are a number of intra-operative factors which influence the likelihood of patients developing PPCs. From a surgical perspective, factors to be considered include surgical approach (open vs. thoracoscopic), duration of operation and post-operative analgesic strategy. A number of anaesthetic factors including ventilatory strategy, intra-operative fluid regime and choice of neuromuscular blocking \& anaesthetic agents must also be considered.

\section{Minimally invasive surgery}

Several studies have assessed the impact of video-assisted thoracoscopic surgery (VATS) on morbidity and mortality after thoracic surgery. A matched case control study of 120 patients undergoing lobectomy demonstrated a significantly lower rate of PORF in the VATS group compared to the open group (1.6\% vs. $8.3 \%)(8)$. This outcome was replicated in another matched case control study of 182 patients, where the rate of PPCs was found to be significantly lower in the VATS group compared to the open group (1.1\% vs. $12.1 \%)(40)$. However, a Danish study of 1,379 patients undergoing lobectomy found (with the exception of pneumonia) no significant difference in the incidence of PPCs between the VATS and open groups (41). 


\section{Anaesthetic agents: TIVA \& NMBDs}

The impact of anaesthetic agents on outcomes after thoracic surgery has also been assessed. A prospective randomised multicentre trial undertaken in Switzerland compared the effects of volatile agents (sevoflurane) and total intravenous anaesthetic (TIVA) agents (propofol). Results demonstrated a greater incidence of PPCs and PORF in the TIVA group, as well as a more pronounced inflammatory response during single lung ventilation, suggesting that volatile anaesthetic agents may confer a protective benefit on patients undergoing thoracic surgery (42). Additional intraoperative drugs thought to have an effect on post-operative respiratory function are neuromuscular blocking drugs (NMBDs). Due to their mechanism of action (inducing paralysis) they impair ventilatory function and hence some studies have shown that prolonged post-operative residual paralysis due to usage of long-acting NMBDs increases the incidence of PPCs (13). However, these results have not been replicated in other studies and there is currently insufficient evidence to advocate the use of one anaesthetic agent over another.

\section{Duration of anaesthesia and impact of one lung ventilation}

Whilst one lung ventilation is a well-established technique which provides clear benefits for the operating surgeon, associated hypoxia and ischaemia (secondary to prolonged atelectasis and associated hypoxic vasoconstriction) and a subsequent deleterious hypoxia-reoxygenation phenomenon upon re-inflation are well described. Moreover, manipulation of the lung on the operative side is thought to contribute to the inflammatory response seen after thoracic surgery. Therefore, both the operated lung (through atelectrauma and surgeon handling) and the non-operated lung (through barotrauma and volutrauma) are susceptible to damage. It is this damage which can lead to acute lung injury and PORF, as a result of the pathological inflammatory processes that occur as a result of this damage (43).

Prolonged operation time further exacerbates these processes and hence should be considered a risk factor for adverse outcomes. Pei et al. identified operation time in excess of 180 minutes as strongly predictive for postoperative complications on both univariate and multivariate analysis (8).

\section{Ventilatory strategy: the impact of lung protective ventilation (LPV)}

Ventilatory parameters such as tidal volume, airway pressure and positive end expiratory pressure (PEEP) are recognised as important factors in the development of ventilator-induced lung injury (VLI) and many studies have been conducted in an attempt to determine optimal strategy during mechanical ventilation (44). Whilst barotrauma (affecting the ventilated lung) is thought to be the key factor associated with adverse outcomes, additional pathological processes including atelectrauma (affecting the non-ventilated lung) and volutrauma (affecting the ventilated lung) have also been shown to increase the rate of PPCs. The demonstration of high driving pressure (peak inspiratory minus peak end expiratory pressure) as an independent risk factor for PPCs highlights the importance of maintaining airway volumes and pressures within an acceptable range in order to maintain adequate oxygenation and gaseous exchange throughout the period of mechanical ventilation. In the context of single lung ventilation, a 2019 meta-analysis (45) identified 9 studies comprising 838 patients separated into a control group (conventional ventilation) and a lung-protective ventilation (LPV) group. The LPV protocol included low tidal volumes and PEEP. Results demonstrated a significant decrease in acute lung injury and a non-significant decrease in atelectasis and pneumonia in the LPV group. Additional studies have found that low tidal volumes alone (46) and in conjunction with PEEP (47) are significantly associated with a decreased incidence of PPCs. Recruitment manoeuvres are also thought to be beneficial but have not been assessed separately from PEEP.

\section{Spontaneous ventilation}

In order to try and reduce or avoid some of the deleterious effects associated with general anaesthesia, there have been a number of observational studies (predominately single centre) published assessing the feasibility of undertaking awake thoracic surgery (ATS) with spontaneous ventilation. Initial results are promising, with results favouring ATS patients. The largest study to date, a randomised trial of 354 patients undergoing either ATS or intubated VATS thoracic surgery, demonstrated a significant reduction in the incidence of PPCs in the ATS group (4.2\% vs. $10 \%$ 
respectively) (48). Further high-quality evidence is required in this area before firm conclusions can be reached.

\section{Fluid regime}

Research into the pathological processes associated with respiratory failure have identified liberal fluid administration as a factor associated with poor outcomes. Indeed, a number of studies have demonstrated that the combination of inflammatory processes (triggered by ventilation of the nonoperated lung and manipulation of the operated lung) and a liberal fluid regime (particularly in those patients who have undergone an extensive resection) significantly increases the likelihood of patients developing PPCs, particularly ARDS (49-51). Overall conclusions from evidence in this area suggest that maintenance of euvolaemia should be the approach of choice, since if fluid administration is too restricted, the ensuing hypovolaemia can affect renal function.

\section{The role of the right heart}

A more recent finding is the phenomenon of right ventricular (RV) dysfunction after lung resection. This finding from McCall et al. has been further studied by assessing right heart function with both transthoracic echocardiography (TTE) and cardiac magnetic resonance (CMR) imaging (52). However, there are no highquality trials assessing the impact of this important postoperative finding on clinical outcomes. One small trial, reviewing post-operative morbidity in patients undergoing pneumonectomy, found RV function to be strongly associated with long-term cardiopulmonary disability (53). Additional studies have shown a relationship between cardiovascular complications and RV dysfunction, but no studies have focussed specifically on the relationship between RV dysfunction and PPCs.

\section{Post-operative analgesia}

Rib injury, disruption of the intercostal neurovascular bundle and intrapleural irritation secondary to chest drain placement are among the many reasons why patients often experience significant pain and discomfort after undergoing thoracic surgery. Analgesic strategies for patients undergoing thoracic surgery have evolved over the years. Whilst thoracic epidural anaesthesia was traditionally used for this patient group, paravertebral blocks and epipleural catheters have found increased favour in more recent times. Prolonged adequate analgesia is crucial in this cohort, and hence the utilization of a catheter technique (whether epipleural or epidural) is preferred over a single-shot analgesia technique in order to avoid rebound pain. In the absence of adequate analgesia, patients will be reluctant to cough and clear secretions, (which can lead to pneumonia) or breathe deeply (which can cause hypoxia secondary to splinting of the diaphragm). Current guidelines recommend pre-emptive and multimodal analgesia throughout the postoperative period and beyond (19).

\section{Post-operative mobilisation}

When the impact of early post-operative mobilisation [which is the founding principle upon which Enhanced Recovery After Surgery (ERAS) programmes were established] on outcomes after thoracic surgery was examined, there was found to be extremely limited evidence to support the role of early mobilisation in reducing PPCs. Nevertheless, given the intuitive benefits of promoting early mobilisation and the low degree of potential patient harm associated with the intervention, the ESTS-ERASS group strongly recommended continuing to encourage early postoperative mobilisation (19).

\section{Post-operative incentive spirometry and physiotherapy}

Incentive spirometry is incorporated into the postoperative physiotherapy regime for patients after thoracic surgery. Whilst some studies have demonstrated that their usage leads to an increase in predicted post-operative predicted FEV1 values (54), none of the research published in this area has been able to demonstrate that the use of incentive spirometry affects clinical outcomes $(55,56)$.

Again, the benefit of post-operative physiotherapy after thoracic surgery seems intuitive. However, as with other interventions such as early mobilisation and incentive spirometry, there is no high-quality evidence available to support its use or to demonstrate a reduction in mortality or PPCs. Whilst one paper did show a significantly reduced rate of atelectasis in those patients receiving post-operative physiotherapy (57), this benefit did not extend to PPCs as a whole. A number of other papers investigating the impact of physiotherapy failed to demonstrate any benefit in terms of PPCs or mortality (58-60). 


\section{Management of postoperative respiratory failure}

Management of PORF should begin with simple interventions such as administration of oxygen therapy, physiotherapy and antimicrobials where appropriate. In those patients with more severe PORF, continuous positive airway pressure or high-flow nasal oxygen (HFNO) therapy should be considered. HFNO is felt to be superior to conventional oxygen therapy, as it tends to be more welltolerated and has the added benefit of delivering a small amount of PEEP in addition to oxygen therapy which may have a positive effect on those patients whose PORF is due to atelectasis. Some studies have demonstrated a reduced rate of re-intubation and increased overall survival when comparing HFNO to regular oxygen therapy. However other studies have not been able to demonstrate a significant difference between the two treatment modalities (61). In those patients with lobar collapse and/or significant sputum retention, intervention with bronchoscopy can be of benefit. Research focussing specifically on the management of ARDS is also available: these studies advocate LPV, cautious fluid administration and placing the patient in the prone position (43).

Auriant et al. (62) assessed the impact of non-invasive positive pressure ventilation (NPPV) in patients with acute hypoxaemic respiratory insufficiency (AHRI) after thoracic surgery, the first such study to do so. They defined AHRI as patients with at least three of the following criteria: respiratory rate of at least 25 breaths/minute, active contraction of accessory respiratory muscles, arterial $\mathrm{PaO}_{2}: \mathrm{FiO}_{2}$ ratio $<200$ and chest radiograph abnormalities (alveolar condensation, atelectasis or pulmonary oedema). Forty-eight patients developed AHRI during the study period and were randomised to NPPV or non-NPPV management. The proportion of patients requiring intubation was significantly less in the NPPV group ( $20.8 \%$ vs. $50 \%, \mathrm{P}=0.035)$. In-hospital mortality was also significantly reduced in the NPPV group $(12.5 \%$ vs. $37.5 \%$, $\mathrm{P}=0.045$ ).

Despite the small number of patients included, and the adoption of an alternative definition of respiratory failure, this study strongly suggests that early use of NPPV in patients with PORF can reduce the need for intubation and improve survival. Indeed, early identification and treatment of the patient with PORF is crucial, in order to prevent further deterioration, as many studies have demonstrated mortality in excess of $65 \%$ amongst patients requiring reintubation after thoracic surgery $(62,63)$.

\section{Postoperative use of VV-ECMO}

Veno-venous extra-corporeal membrane oxygenation ( VVECMO) can be used in the management of severe refractory respiratory failure and provides respiratory support alone: if there is haemodynamic compromise in addition to respiratory failure, veno-arterial ECMO (V-A ECMO) is usually considered a more appropriate therapy, due to its ability to provide concurrent cardiorespiratory support in the acutely unwell patient. V-V ECMO is established via a pipe inserted percutaneously into the femoral vein and advanced into the hepatic inferior vena cava (IVC). Deoxygenated blood is drained from the IVC via this pipe, passed through the oxygenator, and subsequently delivered back into the body through a pipe which has been inserted percutaneously into the right internal jugular vein and advanced to a position where the superior vena cava meets the right atrium (SVC-RA junction) (64).

Whilst a number of papers have examined the outcomes of patients with ARDS who have been subsequently established on VV-ECMO, very few published papers specifically consider patients established on VV-ECMO for respiratory failure after thoracic surgery. A small study of patients undergoing pneumonectomy identified 8 patients requiring $\mathrm{VV}$-ECMO for refractory ARDS and published favourable outcomes (65). However, there is currently no large-volume data available demonstrating outcome in this patient population.

\section{Conclusions}

Table 1 provides an overall summary of the interventions that we have recommended throughout this review as strategies to reduce the incidence of PORF. PPCs are common after thoracic surgery and are associated with increased morbidity and mortality, increased post-operative length of stay and healthcare costs and decreased patient satisfaction. Careful patient selection is imperative to identify prohibitively high-risk patients; however, it is recognised that this is not straightforward. All patients accepted for surgery should be reviewed pre-operatively to ensure that all modifiable risk factors have been optimised in a timely fashion. Avoidance of PPCs requires a multidisciplinary approach with pre-operative, intra-operative and post-operative input from a team including surgeons, anaesthetists, nurses and physiotherapists. Whilst there is limited guidance available regarding the management of PORF, the use of NPPV has shown promise. Management of PORF should 
Table 1 Summary of strategies designed to avoid PORF

Summary of best practice

A 75-year-old female with biopsy-proven NSCLC is referred for consideration of surgical resection. Radiological staging is T1aNOM0. She has mild exertional shortness of breath, COPD, hypertension and type 2 diabetes mellitus. She is an ex-smoker who stopped 5 years ago. Her body mass index (BMI) is $17 \mathrm{~kg} / \mathrm{m}^{2}$

Pre-operative management

(I) The patient should undergo pulmonary function tests including spirometry and gas transfer. Predicted post-operative values should also be calculated

(II) Objective functional testing, such as a shuttle walk, should be undertaken

(III) A risk stratification tool, such as Thoracoscore, should be used to calculate the predicted risk of perioperative mortality

(IV) If any of these results, in combination with the pre-existing patient co-morbidities, raise concerns about fitness for surgery, the patient should be discussed at a high-risk multidisciplinary team meeting and/or reviewed in the outpatient clinic by a consultant anaesthetist

(V) The benefits and limitations of alternative treatment modalities, such as chemotherapy and radiotherapy, in comparison to surgical resection, should be discussed with the patient

(VI) A programme of "prehabilitation" should be considered prior to surgery

(VII) Consider review by a respiratory physician to ensure that her COPD treatment regimen is optimised

(VIII) High-calorie supplement drinks should be offered to the patient to try and improve her nutritional status and increase her BMI

(IX) Pre-operative blood tests should be performed. If the patient is found to be anaemic, this should be corrected with intravenous iron supplementation

Intra-operative management

(I) As this is an early stage lung cancer, the surgeon should aim to undertake the lung resection via a minimally invasive video-assisted thoracoscopic approach

(II) Avoidance of long-acting neuromuscular blocking drugs should be considered

(III) Lung protective ventilation strategies (low tidal volumes and the use of PEEP) should be employed to reduce the risk of lung injury

(IV) Intravenous fluid should be administered in order to maintain euvolaemia

Post-operative management

(I) Multimodal analgesia, including short \& long-acting oral analgesics, regional blocks and an epipleural catheter, should be provided

(II) Patients should be encouraged to mobilise early after surgery and engage with physiotherapists in the early post-operative period

(III) The use of incentive spirometry should be considered

Management of PORF

(I) Oxygen therapy should be initiated as required and early use of high-flow nasal oxygen should be considered

(II) Antimicrobials should be prescribed where appropriate

(III) Non-invasive positive pressure ventilation should be considered for the patient with worsening PORF

(IV) In patients with severe refractory respiratory failure, discussion with specialist centres for consideration of VV-ECMO could be considered

NSCLC, non-small cell lung cancer; COPD, chronic obstructive pulmonary disease; PEEP, positive end expiratory pressure; PORF, postoperative respiratory failure; VV-ECMO, veno-venous extracorporeal membrane oxygenation.

focus on prevention of the need for reintubation, which is associated with a catastrophic rate of mortality. In the current era, for patients with refractory PORF despite mechanical ventilation and optimal medical treatment, VVECMO is now a potential additional treatment option for the management of PORF when undertaken by experienced 
clinicians in select centres.

\section{Acknowledgments}

Funding: None.

\section{Footnote}

Provenance and Peer Review: This article was commissioned by the Guest Editor (David Waller) for the series "Complications of Thoracic Surgery-Aetiology, Management and Prevention", published in Shanghai Chest. This article has undergone external peer review.

Conflicts of Interest: All authors have completed the ICMJE uniform disclosure form (available at http://dx.doi. org/10.21037/shc.2020.03.10). The series "Complications of Thoracic Surgery-Aetiology, Management and Prevention" was commissioned by the editorial office without any funding or sponsorship. The authors have no other conflicts of interest to declare.

Ethical Statement: The authors are accountable for all aspects of the work in ensuring that questions related to the accuracy or integrity of any part of the work are appropriately investigated and resolved.

Open Access Statement: This is an Open Access article distributed in accordance with the Creative Commons Attribution-NonCommercial-NoDerivs 4.0 International License (CC BY-NC-ND 4.0), which permits the noncommercial replication and distribution of the article with the strict proviso that no changes or edits are made and the original work is properly cited (including links to both the formal publication through the relevant DOI and the license). See: https://creativecommons.org/licenses/by-nc-nd/4.0/.

\section{References}

1. NCRAS. National Cancer Registration \& Analysis Service and Cancer Research UK: "Chemotherapy, Radiotherapy and Tumour Resections in England: 20132014" workbook 2017.

2. SCTS. The third national thoracic surgery activity and outcomes report 2018.

3. Grigor EJM, Ivanovic J, Anstee C, et al. Impact of Adverse Events and Length of Stay on Patient Experience After Lung Cancer Resection. Ann Thorac Surg
2017;104:382-8.

4. Takamochi K, Oh S, Matsuoka J, et al. Risk factors for morbidity after pulmonary resection for lung cancer in younger and elderly patients. Interact Cardiovasc Thorac Surg 2011;12:739-43.

5. Berry MF, Hanna J, Tong BC, et al. Risk Factors for Morbidity After Lobectomy for Lung Cancer in Elderly Patients. Ann Thorac Surg 2009;88:1093-9.

6. Kim HJ, Cha SI, Kim CH, et al. Risk factors of postoperative acute lung injury following lobectomy for non-small cell lung cancer. Medicine (Baltimore) 2019;98:e15078.

7. Lugg ST, Agostini PJ, Tikka T, et al. Long-term impact of developing a postoperative pulmonary complication after lung surgery. Thorax 2016;71:171-6.

8. Pei G, Zhou S, Han Y, et al. Risk factors for postoperative complications after lung resection for non-small cell lung cancer in elderly patients at a single institution in China. J Thorac Dis 2014;6:1230-8.

9. Garner M, Routledge T, King JE, et al. New-onset atrial fibrillation after anatomic lung resection: Predictive factors, treatment and follow-up in a UK thoracic centre. Interact Cardiovasc Thorac Surg 2017;24:260-4.

10. De Decker K, Jorens PG, Van Schil P. Cardiac complications after noncardiac thoracic surgery: An evidence-based current review. Ann Thorac Surg 2003;75:1340-8.

11. Ishikawa S, Griesdale DEG, Lohser J. Acute kidney injury after lung resection surgery: Incidence and perioperative risk factors. Anesth Analg 2012;114:1256-62.

12. Abbott TEF, Fowler AJ, Pelosi P, et al. A systematic review and consensus definitions for standardised end-points in perioperative medicine: pulmonary complications. Br J Anaesth 2018;120:1066-79.

13. Miskovic A, Lumb AB. Postoperative pulmonary complications. Br J Anaesth 2017;118:317-34.

14. Radu DM, Jauréguy F, Seguin A, et al. Postoperative Pneumonia After Major Pulmonary Resections: An Unsolved Problem in Thoracic Surgery. Ann Thorac Surg 2007;84:1669-73.

15. Shelley BG, McCall PJ, Glass A, et al. Association between anaesthetic technique and unplanned admission to intensive care after thoracic lung resection surgery: the second Association of Cardiothoracic Anaesthesia and Critical Care (ACTACC) National Audit. Anaesthesia 2019;74:1121-9.

16. McCall PJ, Macfie A, Kinsella J, et al. Critical care after lung resection: CALoR 1, a single-centre pilot study. 
Anaesthesia 2015;70:1382-9.

17. Nicholson A, Lowe MC, Parker J, et al. Systematic review and meta-analysis of enhanced recovery programmes in surgical patients. Br J Surg 2014;101:172-88.

18. Jones NL, Edmonds L, Ghosh S, et al. A review of enhanced recovery for thoracic anaesthesia and surgery. Anaesthesia 2013;68:179-89.

19. Batchelor TJP, Rasburn NJ, Abdelnour-Berchtold E, et al. Guidelines for enhanced recovery after lung surgery: Recommendations of the Enhanced Recovery after Surgery (ERAS@) Society and the European Society of Thoracic Surgeons (ESTS). Eur J Cardiothorac Surg 2019;55:91-115.

20. Al-Sahaf M, Lim E. The association between surgical volume, survival and quality of care. J Thorac Dis 2015;7:S152-5.

21. Lüchtenborg M, Riaz SP, Coupland VH, et al. High procedure volume is strongly associated with improved survival after lung cancer surgery. J Clin Oncol 2013;31:3141-6.

22. Bradley A, Marshall A, Abdelaziz M, et al. Thoracoscore fails to predict complications following elective lung resection. Eur Respir J 2012;40:1496-501.

23. Nwaejike N, Elbur E, Malagon I, et al. Is there a role for the high-risk multidisciplinary team meeting in thoracic surgery? Interact Cardiovasc Thorac Surg 2016;22:397-400.

24. Agostini P, Cieslik H, Rathinam S, et al. Postoperative pulmonary complications following thoracic surgery: Are there any modifiable risk factors? Thorax 2010;65:815-8.

25. Ferguson MK, Lehman AG, Bolliger CT, et al. The Role of Diffusing Capacity and Exercise Tests. Thorac Surg Clin 2008;18:9-17.

26. Brunelli A, Refai MA, Salati M, et al. Carbon monoxide lung diffusion capacity improves risk stratification in patients without airflow limitation: Evidence for systematic measurement before lung resection. Eur J Cardiothorac Surg 2006;29:567-70.

27. Fennelly J, Potter L, Pompili C, et al. Performance in the shuttle walk test is associated with cardiopulmonary complications after lung resections. J Thorac Dis 2017;9:789-95.

28. Win T, Jackson A, Groves AM, et al. Relationship of shuttle walk test and lung cancer surgical outcome. Eur J Cardiothorac Surg 2004;26:1216-9.

29. Benzo RP, Sciurba FC. Oxygen consumption, shuttle walking test and the evaluation of lung resection. Respiration 2010;80:19-23.

30. Miyamoto S, Nagaya N, Satoh T, et al. Clinical correlates and prognostic significance of six-minute walk test in patients with primary pulmonary hypertension: Comparison with cardiopulmonary exercise testing. Am J Respir Crit Care Med 2000;161:487-92.

31. Licker MJ, Widikker I, Robert J, et al. Operative Mortality and Respiratory Complications After Lung Resection for Cancer: Impact of Chronic Obstructive Pulmonary Disease and Time Trends. Ann Thorac Surg 2006;81:1830-7.

32. Brunelli A, Al Refai M, Monteverde M, et al. Stair climbing test predicts cardiopulmonary complications after lung resection. Chest 2002;121:1106-10.

33. Gagné S, McIsaac DI. Modifiable risk factors for patients undergoing lung cancer surgery and their optimization: A review. J Thorac Dis 2018;10:S3761-72.

34. Stéphan F, Boucheseiche S, Hollande J, et al. Pulmonary complications following lung resection: A comprehensive analysis of incidence and possible risk factors. Chest 2000;118:1263-70.

35. Bernard A, Deschamps C, Allen MS, et al. Pneumonectomy for malignant disease: Factors affecting early morbidity and mortality. J Thorac Cardiovasc Surg 2001;121:1076-82.

36. Nakagawa M, Tanaka H, Tsukuma H, et al. Relationship between the duration of the preoperative smoke-free period and the incidence of postoperative pulmonary complications after pulmonary surgery. Chest 2001;120:705-10.

37. Licker M, de Perrot M, Spiliopoulos A, et al. Risk factors for acute lung injury after thoracic surgery for lung cancer. Anesth Analg 2003;97:1558-65.

38. Paull DE, Updyke GM, Davis CA, et al. Complications and long-term survival for alcoholic patients with resectable lung cancer. Am J Surg 2004;188:553-9.

39. Sebio Garcia R, Yáñez Brage MI, Giménez Moolhuyzen $\mathrm{E}$, et al. Functional and postoperative outcomes after preoperative exercise training in patients with lung cancer: a systematic review and meta-analysis. Interact Cardiovasc Thorac Surg 2016;23:486-97.

40. Jeon JH, Kang CH, Kim HS, et al. Video-assisted thoracoscopic lobectomy in non-small-cell lung cancer patients with chronic obstructive pulmonary disease is associated with lower pulmonary complications than open lobectomy: A propensity score-matched analysis. Eur J Cardiothorac Surg 2014;45:640-5.

41. Laursen LØ, Petersen RH, Hansen HJ, et al. Video-assisted thoracoscopic surgery lobectomy for lung cancer is associated with a lower 30-day morbidity compared with lobectomy by thoracotomy. Eur J Cardiothorac Surg 2016;49:870-5. 
42. De Conno E, Steurer MP, Wittlinger M, et al. Anestheticinduced improvement of the inflammatory response to one-lung ventilation. Anesthesiology 2009;110:1316-26.

43. Charlesworth M, Barker JM. Causes and management of surgically induced respiratory syndrome and other acute lung injury. BJU Int 2019. DOI: 10.18591/BJUIK.0703.

44. Beitler JR, Malhotra A, Thompson BT. Ventilator-induced Lung Injury. Clin Chest Med 2016;37:633-46.

45. Hu XY, Du B. Lung-protective ventilation during onelung ventilation: Known knowns, and known unknowns. J Thorac Dis 2019;11:S237-40.

46. Blank RS, Colquhoun DA, Durieux ME, et al. Management of one-lung ventilation: Impact of tidal volume on complications after thoracic surgery. Anesthesiology 2016;124:1286-95.

47. Marret E, Cinotti R, Berard L, et al. Protective ventilation during anaesthesia reduces major postoperative complications after lung cancer surgery: A doubleblind randomised controlled trial. Eur J Anaesthesiol 2018;35:727-35

48. Liu J, Cui F, Li S, et al. Nonintubated video-assisted thoracoscopic surgery under epidural anesthesia compared with conventional anesthetic option: a randomized control study. Surg Innov 2015;22:123-30.

49. Arslantas MK, Kara HV, Tuncer BB, et al. Effect of the amount of intraoperative fluid administration on postoperative pulmonary complications following anatomic lung resections. J Thorac Cardiovasc Surg 2015;149:31420, 321.e1.

50. Brandstrup B, Tønnesen H, Beier-Holgersen R, et al. Effects of Intravenous Fluid Restriction on Postoperative Complications: Comparison of Two Perioperative Fluid Regimens - A Randomized Assessor-Blinded Multicenter Trial. Ann Surg 2003;238:641-8.

51. Alam N, Park BJ, Wilton A, et al. Incidence and Risk Factors for Lung Injury After Lung Cancer Resection. Ann Thorac Surg 2007;84:1085-91.

52. McCall P, Soosay A, Kinsella J, et al. The utility of transthoracic echocardiographic measures of right ventricular systolic function in a lung resection cohort. Echo Res Pract 2019;6:7-15.

53. Lewis JW, Bastanfar M, Gabriel F, et al. Right heart function and prediction of respiratory morbidity in patients undergoing pneumonectomy with moderately severe cardiopulmonary dysfunction. J Thorac Cardiovasc Surg 1994;108:169-75.

54. Weiner P, Man A, Weiner M, et al. The effect of incentive spirometry and inspiratory muscle training on pulmonary function after lung resection. J Thorac Cardiovasc Surg 1997;113:552-7.

55. Agostini P, Naidu B, Cieslik H, et al. Effectiveness of incentive spirometry in patients Following thoracotomy and lung resection including those at high risk for developing pulmonary complications. Thorax 2013;68:580-5.

56. Gosselink R, Schrever K, Cops P, et al. Incentive spirometry does not enhance recovery after thoracic surgery. Crit Care Med 2000;28:679-83.

57. Varela G, Ballesteros E, Jiménez MF, et al. Costeffectiveness analysis of prophylactic respiratory physiotherapy in pulmonary lobectomy. Eur J Cardiothorac Surg 2006;29:216-20.

58. Reeve JC, Nicol K, Stiller K, et al. Does physiotherapy reduce the incidence of postoperative complications in patients following pulmonary resection via thoracotomy? A protocol for a randomised controlled trial. J Cardiothorac Surg 2008;3:48.

59. Reilly JJ Jr. Benefits of aggressive perioperative management in patients undergoing thoracotomy. Chest 1995;107:312S-315S.

60. Doyle RL. Assessing and modifying the risk of postoperative pulmonary complications. Chest 1999;115:77S-81S.

61. Drake MG. High-Flow Nasal Cannula Oxygen in Adults: An Evidence-based Assessment. Ann Am Thorac Soc 2018;15:145-55.

62. Auriant I, Jallot A, Hervé P, et al. Noninvasive ventilation reduces mortality in acute respiratory failure following lung resection. Am J Respir Crit Care Med 2001;164:1231-5.

63. Patel RL, Townsend ER, Fountain SW. Elective pneumonectomy: factors associated with morbidity and operative mortality. Ann Thorac Surg 1992;54:84-8.

64. Reeb J, Olland A, Massard G, et al. Extracorporeal life support in thoracic surgery. Eur J Cardiothorac Surg 2018;53:489-94.

65. Reeb J, Olland A, Pottecher J, et al. Extracorporeal Membrane Oxygenation for Acute Respiratory Distress Syndrome After Pneumonectomy. Ann Thorac Surg 2017;103:881-9.

doi: $10.21037 /$ shc. 2020.03 .10

Cite this article as: Taylor $M$, Charlesworth $M$, Hayes T, Joshi V. Aetiology, management and strategies for prevention of postoperative respiratory failure (PORF) after thoracic surgery. Shanghai Chest 2021;5:5. 